\title{
The safety and cost-analysis of simultaneous versus staged bilateral total knee arthroplasty in a Taiwan population
}

\section{Te-Feng Arthur Chou}

Taipei Veterans General Hospital

Hsuan-Hsiao Ma

Taipei Veterans General Hospital

\section{Yu-Chun Hsu}

Chang Gung Memorial Hospital

\section{Chi-Wu Tsai}

Taipei Veterans General Hospital

Shang-Wen Tsai ( $\nabla$ swtsai.vghtpe@gmail.com )

Taipei Veterans General Hospital

\section{Cheng-Fong Chen}

Taipei Veterans General Hospital

\section{Fang-Yao Chiu}

Taipei Veterans General Hospital

\section{Po-Kuei Wu}

Taipei Veterans General Hospital

\section{Tain-Hsiung Chen}

Taipei Veterans General Hospital

\section{Wei-Ming Chen}

Taipei Veterans General Hospital

\section{Research Article}

Keywords: Bilateral total knee arthroplasty, complication, cost-effective analysis, simultaneous, staged, Taiwan

Posted Date: January 6th, 2022

DOI: https://doi.org/10.21203/rs.3.rs-1186553/v1

License: (c) (1) This work is licensed under a Creative Commons Attribution 4.0 International License. Read Full License 


\section{Abstract}

The purpose of this study was to investigate the safety of Simultaneous, bilateral TKA (SiTKA). Furthermore, we also assessed the cost reduction of SiTKA in comparison with Staged, bilateral TKA (StTKA). We retrospectively review all patients that underwent SiTKA or StTKA due to osteoarthritis (OA) or spontaneous osteonecrosis of the knee (SONK).We assessed length of stay, transfusion rate, early postoperative complications, 30-day and 90-day readmission rate, 1-year reoperation rate and the indication for reoperation. Furthermore, we analyzed the total cost of the two groups, reimbursement from the national health insurance $(\mathrm{NHI})$, cost of the procedures, and net income from each case. A total of 2016 patients (1565 SiTKA and 451 StTKAs) were included in this study. There were no significant differences in terms of complication rates, 30-day and 90-day readmission, and 1-year reoperations between the two groups. The total length of stay was on average 5.0 days longer for StTKA $(p<0.01)$. In terms of cost, all categories of medical costs were significantly lower in SiTKA, while the net hospital income was significantly higher for StTKA. In conclusion, SiTKA and StTKA have similar postoperative complication, readmission and reoperation rates, while SiTKA significantly reduces medical expenses for the patient and $\mathrm{NHI}$.

Level of evidence: level III, retrospective cohort study

\section{Introduction}

Osteoarthritis (OA) is the most common musculoskeletal disorder and affects over 303 million people worldwide.[15] In particular, OA of the knee is a progressive joint disease characterized by chronic pain and functional impairment.[15] When conservative treatment has failed, total knee arthroplasty (TKA) is a common surgical procedure to treat severe knee OA.[1] Primary, unilateral TKAs have been shown to be a cost-effective surgery that relieves pain and improves quality of life for patients with severe OA.[13, 17, 21] Interestingly, for patients that underwent an unilateral TKA, 37\% will receive a TKA for the contralateral knee.[20] Therefore, when OA is present in bilateral knees, bilateral TKAs may be warranted. $[1,11,21]$ The decision to perform simultaneous (SiTKA) or staged TKA (StTKA) for severe OA of bilateral knees remains controversial. $[1,11,24]$ In a meta-analysis performed by Restrepo et al., the authors concluded that SiTKA carries a higher risk for serious cardiopulmonary complications (eg. gastrointestinal distress, deep vein thrombosis, pulmonary and fat embolism) and mortality.[24] On the other hand, Hadley et al. reviewed 438 patients (371 SiTKA and 67 StTKAs), and concluded that there were no significant differences in terms of postoperative complications but SiTKA was associated with a higher transfusion rate.[11] In a recent comparative study, Alghadir et al. compared the reduction of pain levels and functional recovery after SiTKA with StTKA.[1] At postoperative 30 days, there was no significant difference regarding pain intensity and recovery of function between the groups.[1] The authors further recommended that SiTKA to be performed as oppose to StTKA since it could potentially reduce the cost and rehabilitation process for patients with severe, bilateral knee OA.[1] Currently, most authors have agreed that SiTKA can be performed safely in appropriately selected patients with no significant increase in perioperative morbidities. $[1,11,12]$ In addition to the clinical advantages of 
performing SiTKA, many authors have further evaluated the economic advantages. $[18,21,27]$ In a single institution study performed by Stubbs et al., they noted a cost reduction of 37.5\%.[27] The reduction can be attributed to many reasons such as shorter hospital stays, receiving anesthesia only one time, and staying in the postoperative recovery room only once.[27] Current literature suggests that a $24-43 \%$ of total cost savings for SiTKA can be anticipated when compared with StTKA.[21, 27] Although bilateral TKAs are frequently performed in Taiwan, there are very few reports assessing the perioperative outcome and economic benefits of SiTKA.[4, 16] In this study, we aim to assess the safety of SiTKA and StTKA as well as the economic burden of both procedures. We hypothesize that SiTKA can be performed safely without significant increase in complication and readmission rates, while reducing the total cost for the patient and hospital.

\section{Methods}

This retrospective, cohort study was performed at a single, tertiary medical center in Taipei, Taiwan. The study was performed in accordance with the Declaration of Helsinki. The institutional review board (IRB) of Taipei Veterans General Hospital approved this study (IRB number:2020-03-008CC). From January 2011 through December 2016, we obtained medical records and radiology images from the Big Data Center of Taipei Veterans General Hospital (BDC, VGHTPE). Informed consents were obtained from each patient and/or their legal guardians. We reviewed patients who had underwent SiTKA or StTKA procedures during this period according to Taiwan's National Health Insurance procedure code: "PCS64169B". This procedure code will be recorded twice on the same day in a SiTKA procedure and on two separate days in a StTKA procedure. We included patients who had underwent SiTKA or StTKA for primary knee osteoarthritis (ICD-10-CM code: M17) or spontaneous osteonecrosis of the knee (SONK, ICD10-CM code: M90.55, M90.56). The decision to proceed with SiTKA or StTKA was made by the patient after thorough explanation of the potential risks and benefits. We excluded patients under 20 years of age, patients with inflammatory arthritis, musculoskeletal tumors, and active or with history of knee infections. The primary outcome was to assess the safety (rate of postoperative complications, 30-day and 90-day readmission, and 1-year reoperation) of SiTKA in the Taiwan population. The secondary outcome was to determine the cost reducing benefits of performing SiTKA in comparison with StTKA. Preoperative status of the patient including the patient's age, sex, body mass index, American Society of Anesthesiologists physical status classification were recorded as shown in Table 1. 
Table 1

Patient Demographics

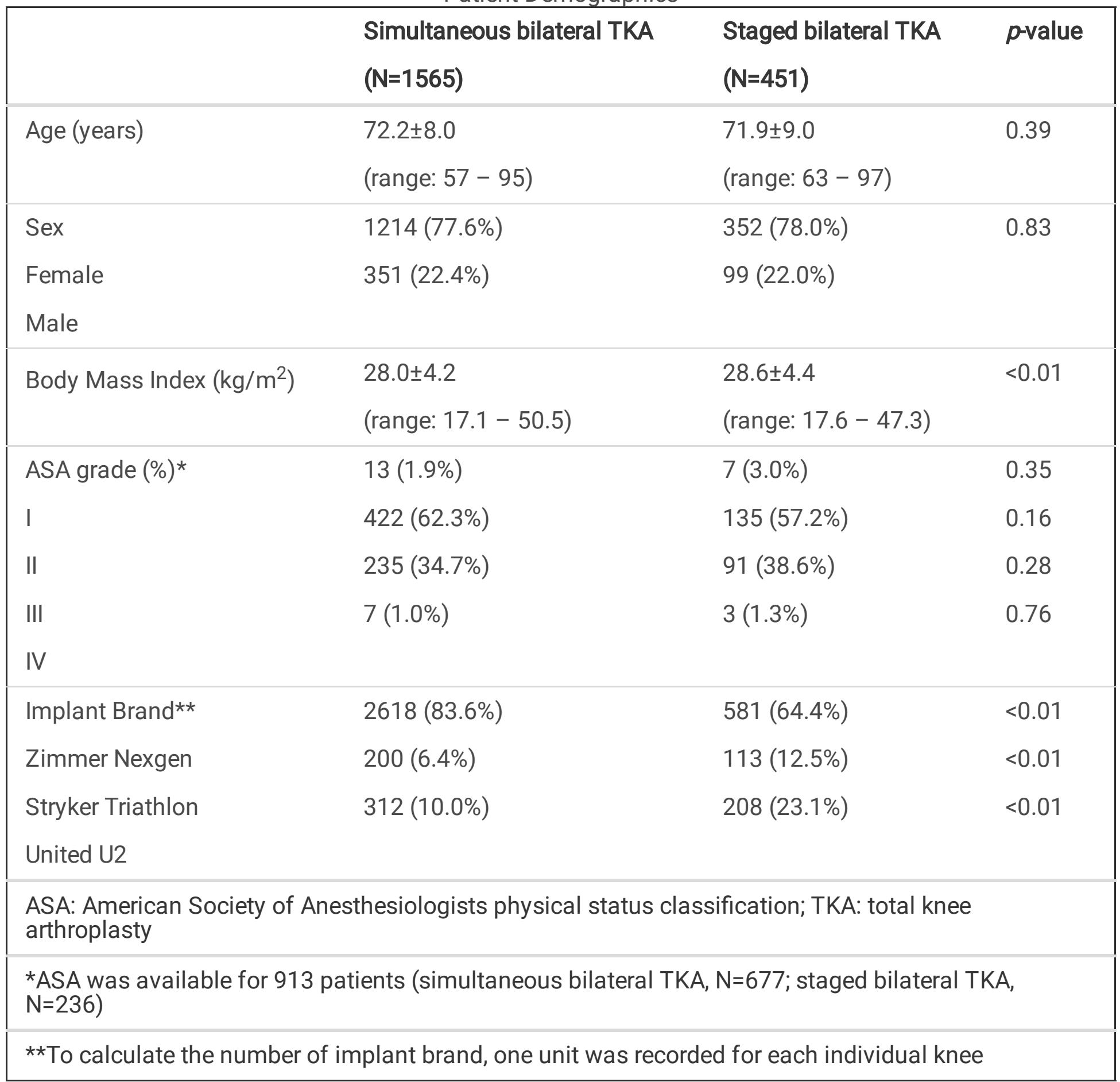

\section{Operative Procedure}

The surgeries were performed by eight, fellowship trained, orthopaedic surgeons. Three types of TKA prosthesis were used, including the NexGen ${ }^{\circledR}$ LPS-Flex knee system (Zimmer Inc., USA), Triathlon ${ }^{\circledR}$ knee system (Stryker, USA), and U2® knee system (United Orthopedic, Taiwan) (Table 1). The patient was first placed in a supine position and bilateral knees were sterilized and draped. A standard mid-vastus approach or medial parapatellar approach was used for all procedures. After arthrotomy and joint exposure, soft tissue dissection and release was completed. The osteophytes were removed and the 
femur and tibia were prepared for resurfacing cuts. For the femur site, the distal femoral cut was performed by intramedullary guide in the alignment at $5-7^{\circ}$ of valgus. Using the Whiteside's line and posterior condyle axis (PCA) as guides, the femoral external rotation was determined. The femoral cuts were then completed based using the cutting guides. For the tibia site, an extramedullary guide was used and a perpendicular cut was made in line with the mechanical axis. Finally, the patella was resurfaced with an onlay technique. The trials for femur, tibia and patella were inserted and appropriate sizing, alignment, tracking and soft tissue balance was achieved. Finally, the implants were inserted and cemented accordingly. The joint capsule was repaired and the subcutaneous tissue and incision were closed accordingly. For patients in SiTKA, the contralateral knee was then performed during the same surgery. The use of closed-suction drain or tourniquet depended on the surgeons' preference.

\section{Postoperative protocols}

All patients adhered to a standard postoperative protocol. We initiated assisted continuous passive motion (CPM) on postoperative day (POD) 1 and weight-bearing as tolerated was recommended for all patients. Each patient received postoperative prophylactic antibiotics (e.g. cefazolin, clindamycin etc.) which was administered intravenously for one day unless there was evidence of infection (eg. postoperative pneumonia, urinary tract infection etc.). The hemoglobin levels were measured on POD1 and if the patient had, 1) hemoglobin level $<9.0 \mathrm{~g} / \mathrm{dL}$, or 2) hemoglobin level between $9.0-10.0 \mathrm{~g} / \mathrm{dL}$ and had symptoms (eg. dizziness, lethargy, pale conjunctiva) suggestive of anemia, 1-2 units of packed red blood cells were transfused. In addition, thromboprophylaxis was given to patients who had a history of thromboembolic diseases, $\mathrm{BMI}>30$, or severe varicose veins. This protocol consisted of an injection of low molecular weight heparin (enoxaparin, Clexane ${ }^{\circledR}, 2000 \mathrm{IU}, 0.2 \mathrm{cc}$ ) immediately after surgery and daily until POD3, and low-dose aspirin (Bokey ${ }^{\circledR}, 100 \mathrm{mg}$ ) for 2 to 5 weeks which was initiated on postoperative day 4. In general, the patient can be discharged once the following criteria have been fulfilled: 1) A dry and clean wound; 2) able to ambulate independently and 3) CPM greater than 90 degrees.

\section{Perioperative outcome}

All medical records of each patient were reviewed by three senior surgeons (TFC, CWT, and SWT). We recorded the patients' length of stay, transfusion rate, early complications within 30 days after the surgery, 30-day and 90-day readmission rate, 1-year reoperation rate and the indication for reoperation. For early complications, we recorded the incidence of venous thromboembolism (deep vein thrombosis and pulmonary embolism), blood transfusion reaction, delirium, fall, periprosthetic fracture, surgical site complications (SSC), periprosthetic joint infections (PJI) and other infection events (e.g. urinary tract infection or pneumonia). Specifically, SSC include superficial wound infections, hematoma and seroma formation that required additional wound care, systemic antibiotics or a surgical procedure. A periprosthetic joint infection (PJI) is a more severe type of infection that involved the bone and joint surface which required extensive debridement or resection of the prosthesis. The patient was considered to have an extensor mechanism failure when the patient is unable to voluntarily extend the knee secondary to a patella fracture, rupture of quadriceps or patellar tendon. Finally, the patient was diagnosed with an unstable protheses if typical symptoms (eg. giving way, initiating pain, difficulty 
climbing stairs, and fear of knee buckling under stress) along with signs such as recurrent joint effusion, joint laxity and a positive stress test were noted upon physical exams.

\section{Medical Cost}

A total of 3 major categories of cost were recorded. All of the medical costs were recorded in New Taiwan Dollars (NTD). The total reimbursement from the national health insurance, the total medical cost for the patient (including clinic visits, admission fee, operating room fee, and anesthesia fee) and the total net income for the hospital were recorded.

\section{Statistical analyses}

All data were entered and analyzed with the SPSS software (version 25.0, SPSS Inc., Chicago, IL). We recorded data as mean, range and standard deviation for continuous variables and the student's t-test was used to compare the differences at appropriate times. For categorical data such as percentages, we used the chi-square and or fisher's exact test when appropriate to assess for statistical significance. A $p$ value $<0.05$ and confidence interval $>95 \%$ was considered to be statistically significant.

\section{Results}

\section{Patient demographics}

After exclusion, a total of 2016 patients were included in this study, with 1565 patients receiving SiTKA and 451 patients with StTKA. The baseline patient demographics are shown in Table 1. The mean age was $72.2 \pm 8.0$ years-old for SiTKA and $71.9 \pm 9.0$ years-old for StTKA $(p>0.05)$. In terms of sex distribution, $77.6 \%$ and $78.0 \%$ were female in SiTKA and StTKA, respectively $(p>0.05)$. The patient's preoperative BMI was $28.0 \pm 4.2 \mathrm{~kg} / \mathrm{m}^{2}$ in SiTKA and $28.6 \pm 4.4 \mathrm{~kg} / \mathrm{m}^{2}$ in StTKA $(p<0.05)$. For patients in SiTKA, $64.2 \%$ were considered to be ASA grade I or II and $35.7 \%$ were considered ASA III or IV. In StTKA, $60.2 \%$ were considered ASA grade I or II, while $39.8 \%$ were ASA grade III or IV. There were no significant differences in terms of ASA grading between the two groups $(p>0.05)$.

\section{Perioperative outcome}

The total length of stay were $6.9 \pm 1.9$ and $11.9 \pm 2.0$ days for SiTKA and StTKA $(p<0.01)$ respectively. The transfusion rate was $89.0 \%$ for SiTKA and $33.5 \%$ for StTKA $(p<0.01)$. In terms of postoperative complications, the most common complication was SSC, followed by PJI and postoperative falls in both groups (Table 2). Both groups had $3.0 \%$ of the patients ( $N=47$ in SiTKA, $N=27$ in StTKA) readmitted within 30 days after the surgery. The 90-day readmission rate for SiTKA was $6.1 \%(\mathrm{~N}=95)$ while StTKA was $6.4 \%$ $(\mathrm{N}=58, \mathrm{p}>0.05)$. The 1 -year reoperation rate was $1.5 \%$ and $0.7 \%(\mathrm{p}>0.05)$ for SiTKA and StTKA, respectively. The most common indication for reoperation were periprosthetic fractures in both groups (Table 2). 
Table 2

Perioperative outcomes

\begin{tabular}{|c|c|c|c|}
\hline & $\begin{array}{l}\text { Simultaneous bilateral } \\
\text { TKA } \\
(n=1565)\end{array}$ & $\begin{array}{l}\text { Staged bilateral } \\
\text { TKA } \\
(n=451)\end{array}$ & $p$-value \\
\hline Length of stay (days) & $\begin{array}{l}6.9 \pm 1.9 \\
\text { (range } 3-46 \text { ) }\end{array}$ & $\begin{array}{l}11.9 \pm 2.0 \\
\text { (range } 3-25 \text { ) }\end{array}$ & $<0.01$ \\
\hline Transfusion rate (\%) & $1393(89.0 \%)$ & $151(33.5 \%)$ & $<0.01$ \\
\hline $\begin{array}{l}\text { Early postoperative complications } \\
(\%) \\
\text { Symptomatic VTE } \\
\text { Severe transfusion reaction } \\
\text { Delirium } \\
\text { Fall } \\
\text { Periprosthetic fracture } \\
\text { Surgical site complication } \\
\text { Periprosthetic joint infection } \\
\text { Others (UTI, pneumonia etc.) }\end{array}$ & $\begin{array}{l}55(3.5 \%) \\
1(0.1 \%) \\
1(0.1 \%) \\
1(0.1 \%) \\
9(0.6 \%) \\
0 \\
25(1.6 \%) \\
9(0.6 \%) \\
9(0.6 \%)\end{array}$ & $\begin{array}{l}23(5.5 \%) \\
1(0.2 \%) \\
0 \\
1(0.2 \%) \\
3(0.7 \%) \\
0 \\
11(2.4 \%) \\
4(0.9 \%) \\
3(0.7 \%)\end{array}$ & 0.12 \\
\hline 30-day readmission & $47(3.0 \%)$ & 27 (3.0\%) & 0.99 \\
\hline 90-day readmission & $95(6.1 \%)$ & $58(6.4 \%)$ & 0.72 \\
\hline 1-year reoperation rate & $23(1.5 \%)$ & $6(0.7 \%)$ & 0.07 \\
\hline Periprosthetic fractures & $11(0.7 \%)$ & $4(0.4 \%)$ & \\
\hline Surgical site complications & $3(0.2 \%$ & 0 & \\
\hline Periprosthetic Joint Infections & $3(0.2 \%)$ & $2(0.2 \%)$ & \\
\hline Extensor mechanism failure & $3(0.2 \%)$ & 0 & \\
\hline Joint instability & $3(0.2 \%)$ & 0 & \\
\hline
\end{tabular}

\section{Medical cost and hospital income}

The total reimbursement from the national health insurance $(\mathrm{NHI})$ was $205526.5 \pm 24177.0$ NTD for SiTKA, and $243463.0 \pm 36271.7$ for StTKA $(p<0.01)$. The total medical cost for the patients were $188888.0 \pm 18960.6$ and $206550.2 \pm 24753.1$ for SiTKA and StTKA, respectively $(p<0.01)$. Specifically, the admission fee, clinic follow-up fee, operating room fee, and anesthesia fee were all significantly lower for 
SiTKA as shown in Table 3. The net hospital income was significantly higher for patients that underwent StTKA $(36912.8 \pm 22895.7)$ when compared with SiTKA $(16638.5 \pm 14434.8, p<0.01)$.

Table 3

Medical cost

\begin{tabular}{|llll|}
\hline & $\begin{array}{l}\text { Simultaneous bilateral TKA } \\
(\mathbf{N}=1565)\end{array}$ & $\begin{array}{l}\text { Staged bilateral TKA } \\
(\mathrm{N}=451)\end{array}$ & $p$-value \\
\hline Polyethylene type in TKA (\%) & $1132(72.3 \%)$ & $335(74.2 \%)$ & 0.41 \\
NHI-covered HXLPE & $242(15.5 \%)$ & $58(12.9 \%)$ & 0.17 \\
Self-paid tibia UHMWPE only & $191(12.2 \%)$ & $58(12.9 \%)$ & 0.71 \\
Self-paid tibia \& patella UHMWPE & & & \\
\hline Total NHI reimbursement (NTD) & $205526.5 \pm 24177.0$ & $243463.0 \pm 36271.7$ & $<0.01$ \\
\hline Total medical cost (NTD) & $188888.0 \pm 18960.6$ & $206550.2 \pm 24753.1$ & $<0.01$ \\
\hline Clinic visits & $2842.6 \pm 628.7$ & $4888.9 \pm 1198.8$ & $<0.01$ \\
\hline Admission fee & $8337.3 \pm 2212.0$ & $14123.4 \pm 3964.3$ & $<0.01$ \\
\hline Operating room fee & $58136.4 \pm 3760.2$ & $59321.0 \pm 6024.0$ & $<0.01$ \\
\hline Anesthesia fee & $5807.5 \pm 2695.2$ & $7047.0 \pm 3563.3$ & $<0.01$ \\
\hline Net hospital income (NTD) & $16638.5 \pm 14434.8$ & $36912.8 \pm 22895.7$ & $<0.01$ \\
\hline NHI: National health insurance; NTD: New Taiwan Dollars & & \\
\hline HXLPE: highly cross-linked polyethylene, UHMWPE: ultra-high molecular weight polyethylene
\end{tabular}

\section{Discussion}

With an aging population, there is an increase demand for TKAs.[15] When patients present with OA of bilateral knees, the decision to perform SiTKA or StTKA remains controversial.[24] The most significant findings of this study was that SiTKA is a safe surgery for patients with advanced OA or SONK of bilateral knees. Moreover, there was a significant cost reduction for the patient and the national health insurance when SiTKA is compared with StTKA. To our knowledge, this was the first cohort study performed in Taiwan to evaluate both the safety and cost reducing benefits of SiTKA.

Some authors have advocated against SiTKA.[24] In an earlier meta-analysis performed by Restrepo et al., the authors concluded there was a higher risk for serious cardiac complications, pulmonary complications and mortality when SiTKA was performed.[24] In our study, the rates for all types of complications (eg. VTE, infection and postoperative delirium) were similar for both groups ( $p>0.05)$. Recent studies have suggested that careful selection of patients can reduce complications to similar rates. $[3,11,27,28]$ For instance, patients that are older and/or have concomitant cardiopulmonary 
conditions, morbidly obese (BMl>40 $\left.\mathrm{kg} / \mathrm{m}^{2}\right)$, and higher ASA scores should be treated with caution due to a relatively higher risk for surgical complications. $[7,9,10,24]$ Interestingly, SiTKA is considered to be a safe procedure in patients with conditions such as rheumatoid arthritis, end-stage hemophilic arthropathy, and moderate obesity (BMI $30-35 \mathrm{~kg} / \mathrm{m}^{2}$ ) which were previously thought as risk factors for postoperative complications in unilateral TKA. $[6,7,14,23,28]$ A recent meta-analysis performed by Fu et al. further concluded that deep infection and revision rates were significantly lower when SiTKA is performed in comparison with staged procedures.[8] In congruent with most of the current literature, the results of this study validated the safety of SiTKA which can be safely performed for most patients. Another concern for bilateral TKAs is the longer hospital stays required for recovery. $[1,24]$ In a national database study performed by Lin et al., the authors noted that patients that received SiTKA were discharged on average, 3 days earlier than patients in the StTKA group $(p<0.05)$. [16] In this study, we also noticed a similar trend in which patients that underwent SiTKA were discharged after $6.9 \pm 1.9$ days, while patients in StTKA stayed for $11.9 \pm 2.0$ days $(p<0.05)$. In addition, increased blood loss is another issue frequently encountered during SiTKA. In this study, $89.0 \%$ of the patients in SiTKA and $33.5 \%$ of the patients in StTKA $(p<0.01)$ received transfusion. This higher incidence for SiTKA could be a result of surgeon preference since many of the surgeons included in this study transfused 1-2 units of packed RBC on a routine basis during SiTKA. However, the number of transfusion reactions between the two groups were similar $(0.6 \%$ vs $0.5 \%, p>0.05)$, further suggesting that although the transfusion rate is increased, the associated morbidity remains that same. In recent reports, the transfusion rate is around $11 \%$ for unilateral TKA, and $28 \%$ for SiTKA. Several studies have discussed methods to reduce transfusion and the morbidities associated with transfusion. In patients with preoperative anemia (eg. $\mathrm{Hb}<10 \mathrm{~g} / \mathrm{L}$ ) complicated with a history of transfusion reactions, autologous blood donation with subsequent reinfusion can be considered.[5] Another option is to use cell salvage modalities (eg. cell saver) to reduce transfusion reactions and the risk for transmission of infections associated with allogeneic blood transfusion.[26] The application of tourniquet and release of tourniquet prior to wound closure to achieve hemostasis has also been shown to effectively reduce intraoperative blood looss.[22] Furthermore, the use of intraoperative tranexamic acid via intravenous or intraarticular route have been also shown to effectively reduce blood loss.[3] In our institution, one or a combination of the above methods are currently in practice and is dependent on the surgeon's preference. Therefore, although there is substantial blood loss and higher rates for transfusion, the overall rate of adverse reactions is minimal.

In our institution, TKAs are performed under either general anesthesia or spinal anesthesia, regardless if its unilateral or bilateral. In addition to the cost reduction and decreased complication rates for only administering anesthesia once, about $3 \%$ of patients will refuse SA for a second surgery.[2] Moreover, $26 \%$ of these patients refused another SA due to fear of back pain.[2] Benzon et al. reported that back pain is a frequent complaint after anesthesia, regardless if its general anesthesia or spinal anesthesia.[2] Therefore, reducing the number of anesthesia procedures not only reduces the costs and risks associated with administering anesthesia, but more importantly patient satisfaction should also improve. Given these advantages, we recommend performing SiTKA as the treatment of choice for most patients that have severe osteoarthritis of bilateral knees. 
Another issue frequently discussed is the cost reduction of SiTKA as the cost for SiTKA has been demonstrated to be significantly lower than staged procedures.[16, 25] In our study, SiTKA had a reduction of $\mathrm{NHI}$ reimbursement of 37936.5 NTD per patient $(p<0.05)$. This reduction also reflected a decreased net income for the hospital (on average -20274.3 NTD). In an earlier study performed in the US, Reuben et al. reported that the total costs reduction of SiTKA can exceed 10,000 USD for each patient when compared with StTKA.[25] In another study performed in Australia by March et al., the authors noted bilateral TKA and unilateral TKA had a similar length of stay in hospital and similar out-of-pocket expenditure.[19] Therefore, with an increasing financial burden due to the increase demand for arthroplasty surgeries, SiTKA appears to be an outstanding procedure for both the patient and the $\mathrm{NHI}$.

This study is not without limitations. The first limitation is that our study was a non-randomized study, retrospective study. Ideally, a randomized, double blinded clinical trial would have had the highest clinical impact. However, due to the nature of the intervention (SiTKA vs. StTKA), it would have been difficult to perform such a study. The second limitation was the lack of patient reported outcome for our study groups. Since our data was obtained from the Big Data Center of Taipei Veterans Hospital, patient reported outcomes were not thoroughly recorded and therefore was not presented in this study. Finally, our institution is a highly specialized, high volume medical center ( $>2000$ primary TKAs per year). Therefore, the data presented may not be applicable to every institution.

\section{Conclusion}

With an increasing demand for TKAs in Taiwan, SiTKA appears to be a safe and beneficial procedure for patients presenting with severe $\mathrm{OA}$ of bilateral knees. In addition, the financial relief provided by performing simultaneous surgeries can further improve patient satisfaction while also reducing the medical expenses of both the patient and the $\mathrm{NHI}$.

\section{Declarations}

Acknowledgements: We do not have any acknowledgements.

Author contributions: TFC collected data, interpreted data, drafted the manuscript and conceptualization of this study. HHM collected, calculated and interpreted data. $\mathrm{YCH}$ assisted with data interpretation and conceptualization of the study. CWT collected and interpreted data. SWT was the primary surgeon, interpreted data, assisted with conceptualizing the study and drafted the manuscript. CFC performed the surgeries and assisted with study design. FYC performed the surgeries. PKW assisted with data interpretation. THC performed some of the surgeries. WMC was the primary surgeon and conceptualized this study.

Competing Interests: The authors have no competing interests

\section{References}


1. Alghadir AH, Iqbal ZA, Anwer S, Anwar D (2020) Comparison of simultaneous bilateral versus unilateral total knee replacement on pain levels and functional recovery. BMC Musculoskelet Disord 21:246

2. Benzon HT, Asher YG, Hartrick CT (2016) Back Pain and Neuraxial Anesthesia. Anesth Analg 122:2047-2058

3. Chen SH, Chen JY, Wong TH, Buechel F, Jr., Wu MH (2020) Simultaneous Bilateral Total Knee Arthroplasty Becomes Safer after Institution of Optimal Perioperative Management: A Longitudinal Cohort Study. J Knee Surg;10.1055/s-0039-1700979

4. Cheng YC, Wu PK, Chen CF, Chen CM, Tsai SW, Chang MC, et al. (2019) Analysis of learning curve of minimally invasive total knee arthroplasty: A single surgeon's experience with 4017 cases over a 9-year period. J Chin Med Assoc 82:576-583

5. Cushner FD, Scott WN, Scuderi G, Hill K, Insall JN (2005) Blood loss and transfusion rates in bilateral total knee arthroplasty. J Knee Surg 18:102-107

6. Danoff JR, Moss G, Liabaud B, Geller JA (2013) Total knee arthroplasty considerations in rheumatoid arthritis. Autoimmune Dis 2013:185340

7. Fabi DW, Mohan V, Goldstein WM, Dunn JH, Murphy BP (2011) Unilateral vs bilateral total knee arthroplasty risk factors increasing morbidity. J Arthroplasty 26:668-673

8. Fu D, Li G, Chen K, Zeng H, Zhang X, Cai Z (2013) Comparison of clinical outcome between simultaneous-bilateral and staged-bilateral total knee arthroplasty: a systematic review of retrospective studies. J Arthroplasty 28:1141-1147

9. Giori NJ, Amanatullah DF, Gupta S, Bowe T, Harris AHS (2018) Risk Reduction Compared with Access to Care: Quantifying the Trade-Off of Enforcing a Body Mass Index Eligibility Criterion for Joint Replacement. J Bone Joint Surg Am 100:539-545

10. Gronbeck C, Cote MP, Lieberman JR, Halawi MJ (2019) Risk stratification in primary total joint arthroplasty: the current state of knowledge. Arthroplast Today 5:126-131

11. Hadley S, Day M, Schwarzkopf R, Smith A, Slover J, Zuckerman J (2017) Is Simultaneous Bilateral Total Knee Arthroplasty (BTKA) as Safe as Staged BTKA? Am J Orthop (Belle Mead NJ) 46:E224-E229

12. Husted H, Troelsen A, Otte KS, Kristensen BB, Holm G, Kehlet H (2011) Fast-track surgery for bilateral total knee replacement. J Bone Joint Surg Br 93:351-356

13. Jenkins PJ, Clement ND, Hamilton DF, Gaston P, Patton JT, Howie CR (2013) Predicting the costeffectiveness of total hip and knee replacement: a health economic analysis. Bone Joint J 95-B:115-121 
14. Jiang C, Zhao Y, Feng B, Zhai J, Bian Y, Qiu G, et al. (2018) Simultaneous bilateral total knee arthroplasty in patients with end-stage hemophilic arthropathy: a mean follow-up of 6 years. Sci Rep 8:1608

15. Kloppenburg M, Berenbaum F (2020) Osteoarthritis year in review 2019: epidemiology and therapy. Osteoarthritis Cartilage 28:242-248

16. Lin AC, Chao E, Yang CM, Wen HC, Ma HL, Lu TC (2014) Costs of staged versus simultaneous bilateral total knee arthroplasty: a population-based study of the Taiwanese National Health Insurance Database. J Orthop Surg Res 9:59

17. Losina E, Walensky RP, Kessler CL, Emrani PS, Reichmann WM, Wright EA, et al. (2009) Costeffectiveness of total knee arthroplasty in the United States: patient risk and hospital volume. Arch Intern Med 169:1113-1121; discussion 1121-1112

18. Macario A, Schilling P, Rubio R, Goodman S (2003) Economics of one-stage versus two-stage bilateral total knee arthroplasties. Clin Orthop Relat Res;10.1097/01.blo.0000079265.91782.ca149-156

19. March LM, Cross M, Tribe KL, Lapsley HM, Courtenay BG, Cross MJ, et al. (2004) Two knees or not two knees? Patient costs and outcomes following bilateral and unilateral total knee joint replacement surgery for OA. Osteoarthritis Cartilage 12:400-408

20. McMahon M, Block JA (2003) The risk of contralateral total knee arthroplasty after knee replacement for osteoarthritis. J Rheumatol 30:1822-1824

21. Odum SM, Troyer JL, Kelly MP, Dedini RD, Bozic KJ (2013) A cost-utility analysis comparing the cost-effectiveness of simultaneous and staged bilateral total knee arthroplasty. J Bone Joint Surg Am 95:1441-1449

22. Prasad N, Padmanabhan V, Mullaji A (2007) Blood loss in total knee arthroplasty: an analysis of risk factors. Int Orthop 31:39-44

23. Radmer S, Andresen R, Sparmann M (2006) [Simultaneous bilateral total knee arthroplasty in patients with rheumatoid arthritis]. Z Orthop Ihre Grenzgeb 144:472-476

24. Restrepo C, Parvizi J, Dietrich T, Einhorn TA (2007) Safety of simultaneous bilateral total knee arthroplasty. A meta-analysis. J Bone Joint Surg Am 89:1220-1226

25. Reuben JD, Meyers SJ, Cox DD, Elliott M, Watson M, Shim SD (1998) Cost comparison between bilateral simultaneous, staged, and unilateral total joint arthroplasty. J Arthroplasty 13:172-179

26. Shenolikar A, Wareham K, Newington D, Thomas D, Hughes J, Downes M (1997) Cell salvage auto transfusion in total knee replacement surgery. Transfus Med 7:277-280 
27. Stubbs G, Pryke SE, Tewari S, Rogers J, Crowe B, Bridgfoot L, et al. (2005) Safety and cost benefits of bilateral total knee replacement in an acute hospital. ANZ J Surg 75:739-746

28. Taylor BC, Dimitris C, Mowbray JG, Gaines ST, Steensen RN (2010) Perioperative safety of twoteam simultaneous bilateral total knee arthroplasty in the obese patient. J Orthop Surg Res 5:38 